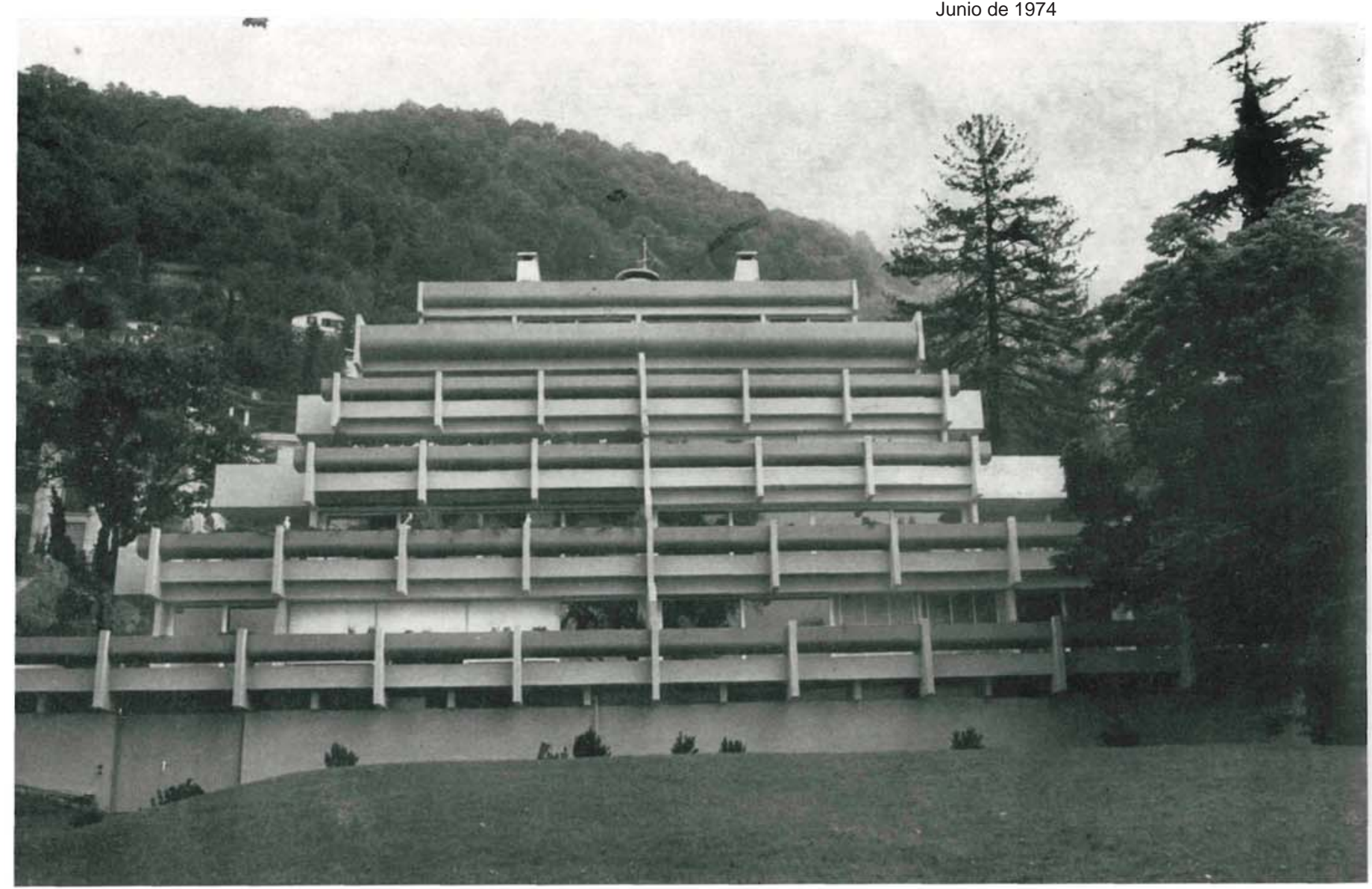

\title{
edificio residencial en Montreux SUIZA
}
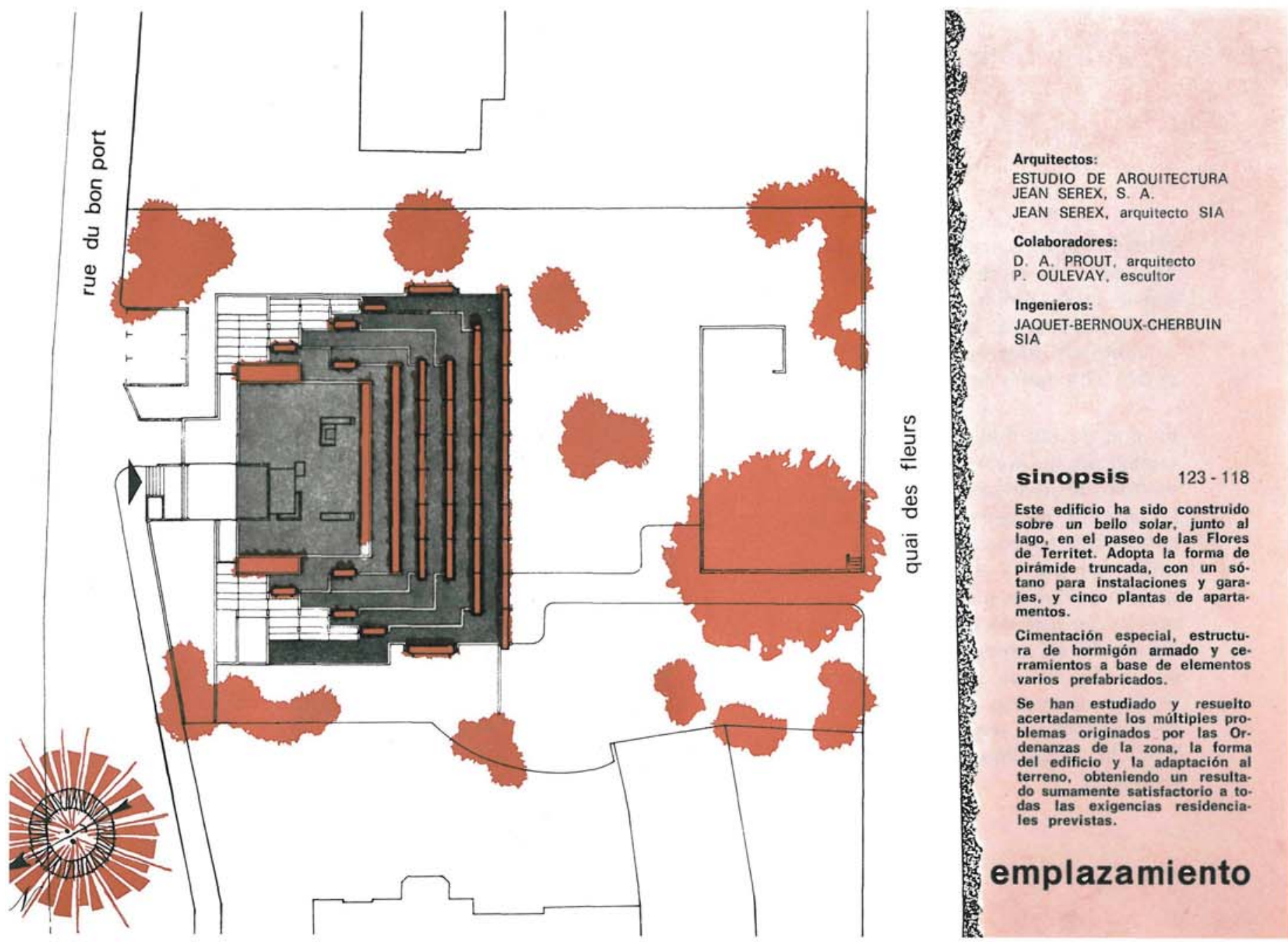


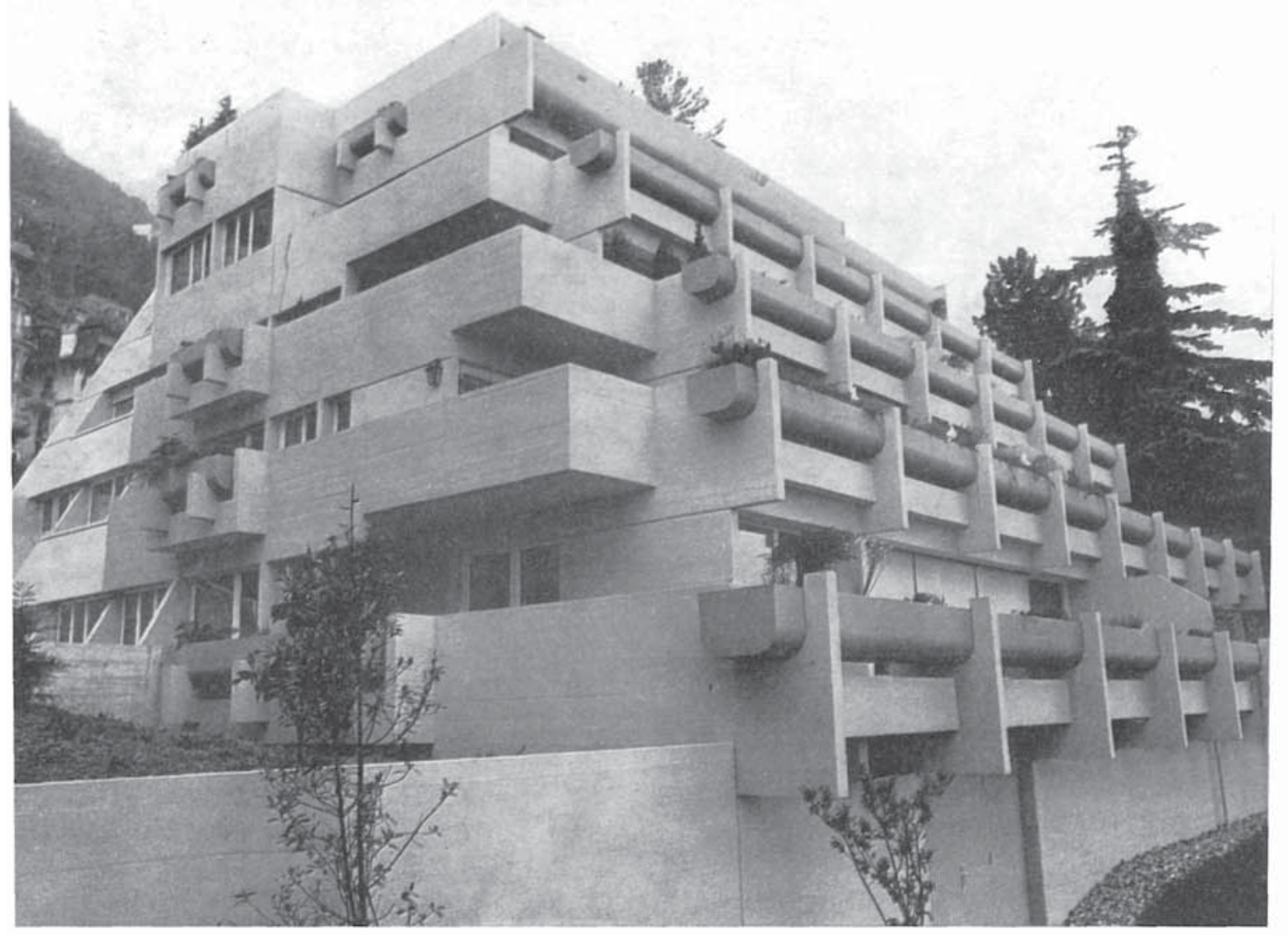

Situado en el extremo de un golfo, adosado a la montaña y bañado por el lago Léman,

Montreux-Territet es un

privilegiado lugar de turismo suizo, con fama internacional.

En una de las mejores zonas de la ciudad se ha levantado este edificio de apartamentos de lujo. La parcela utilizada, de $2.750 \mathrm{~m}^{2}$ de superficie, se encuentra en la orilla del lago y en la proximidad de una pequeña montaña que la protege de los vientos del norte. La construcción ocupa $740 \mathrm{~m}^{2}$ de superficie, en un terreno de fuerte inclinación, dedicándose el resto, más de $2.000 \mathrm{~m}^{2}$, a jardines y zonas arboladas.

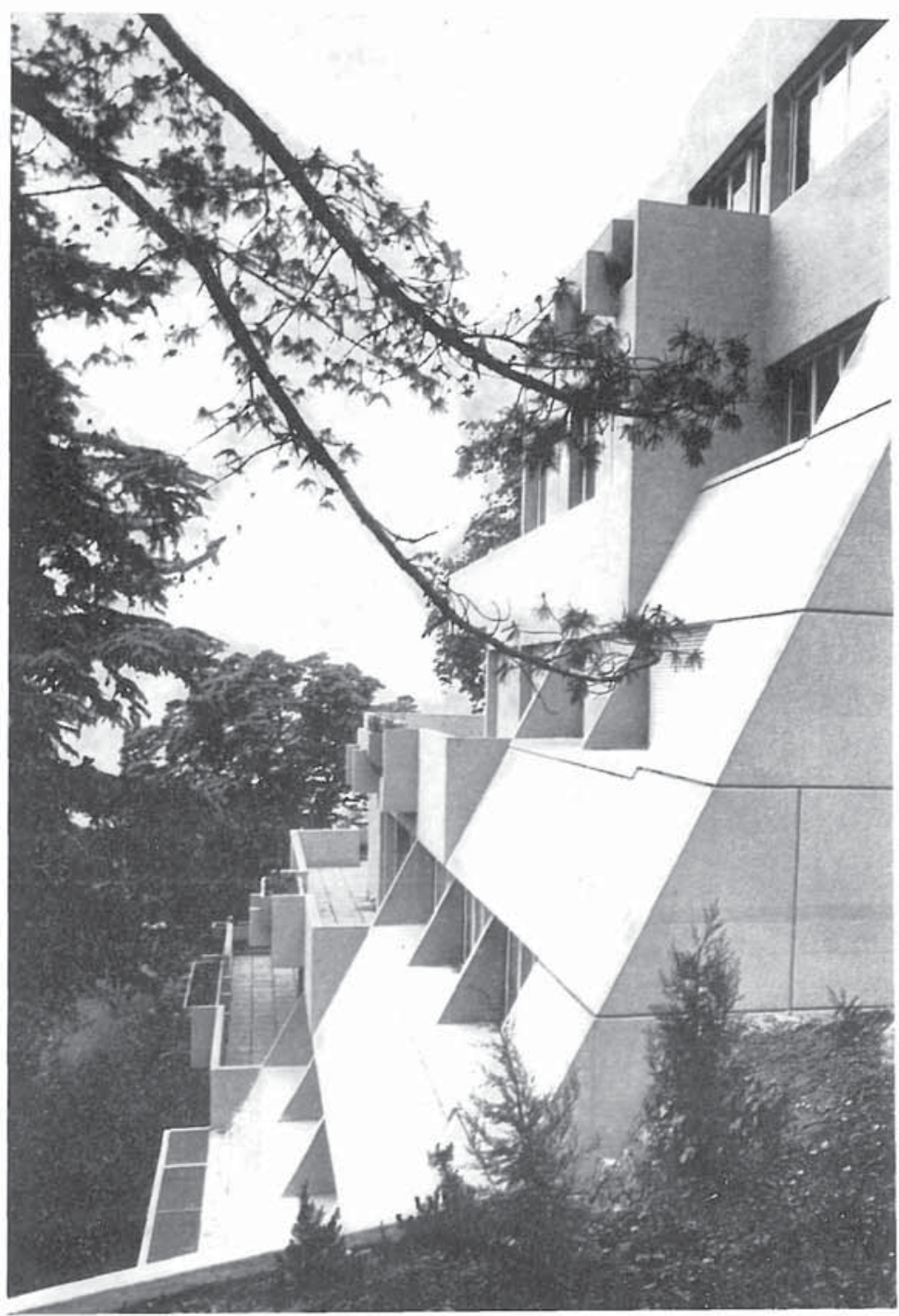



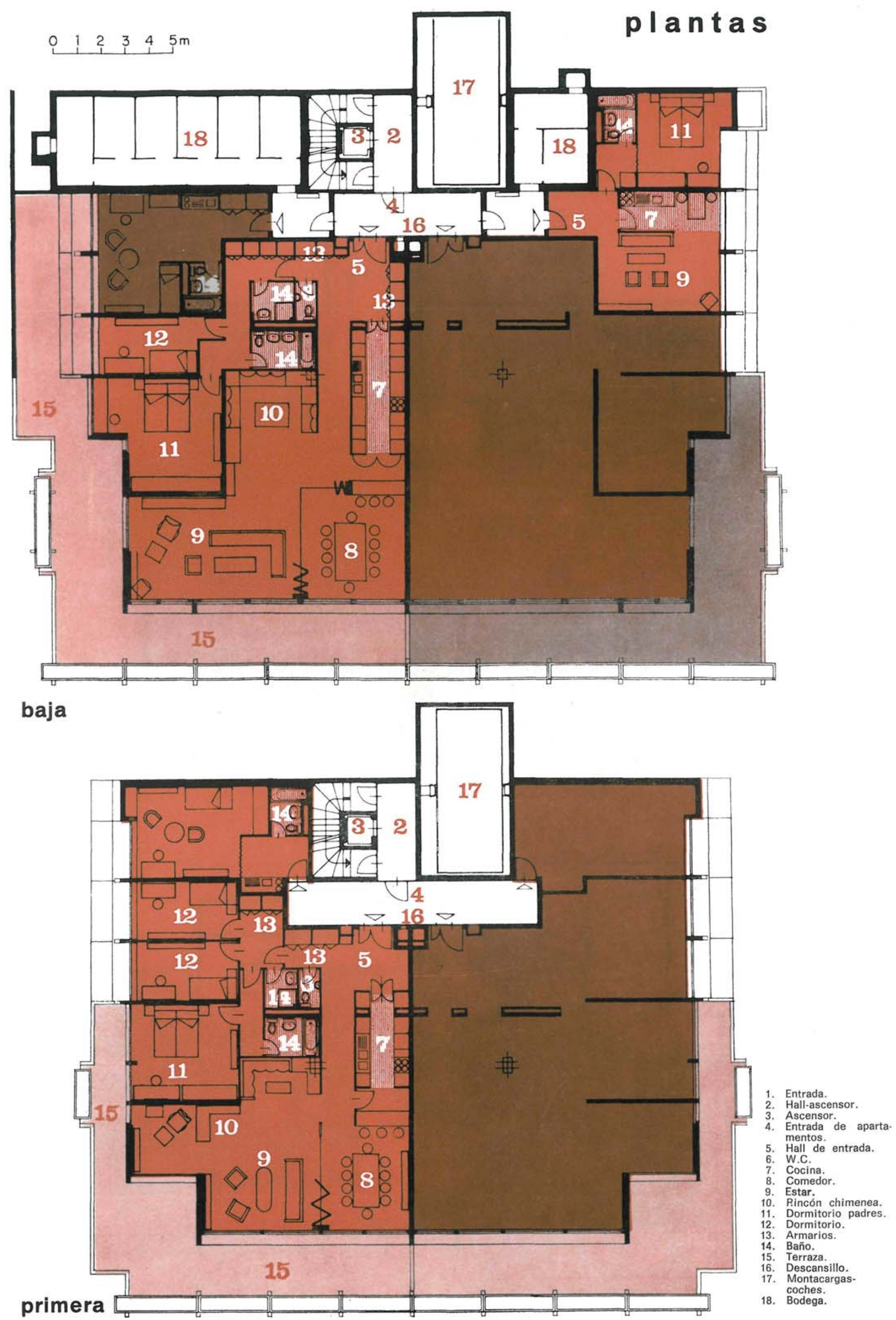

(C) Consejo Superior de Investigaciones Científicas 


\section{plantas}

\section{segunda}

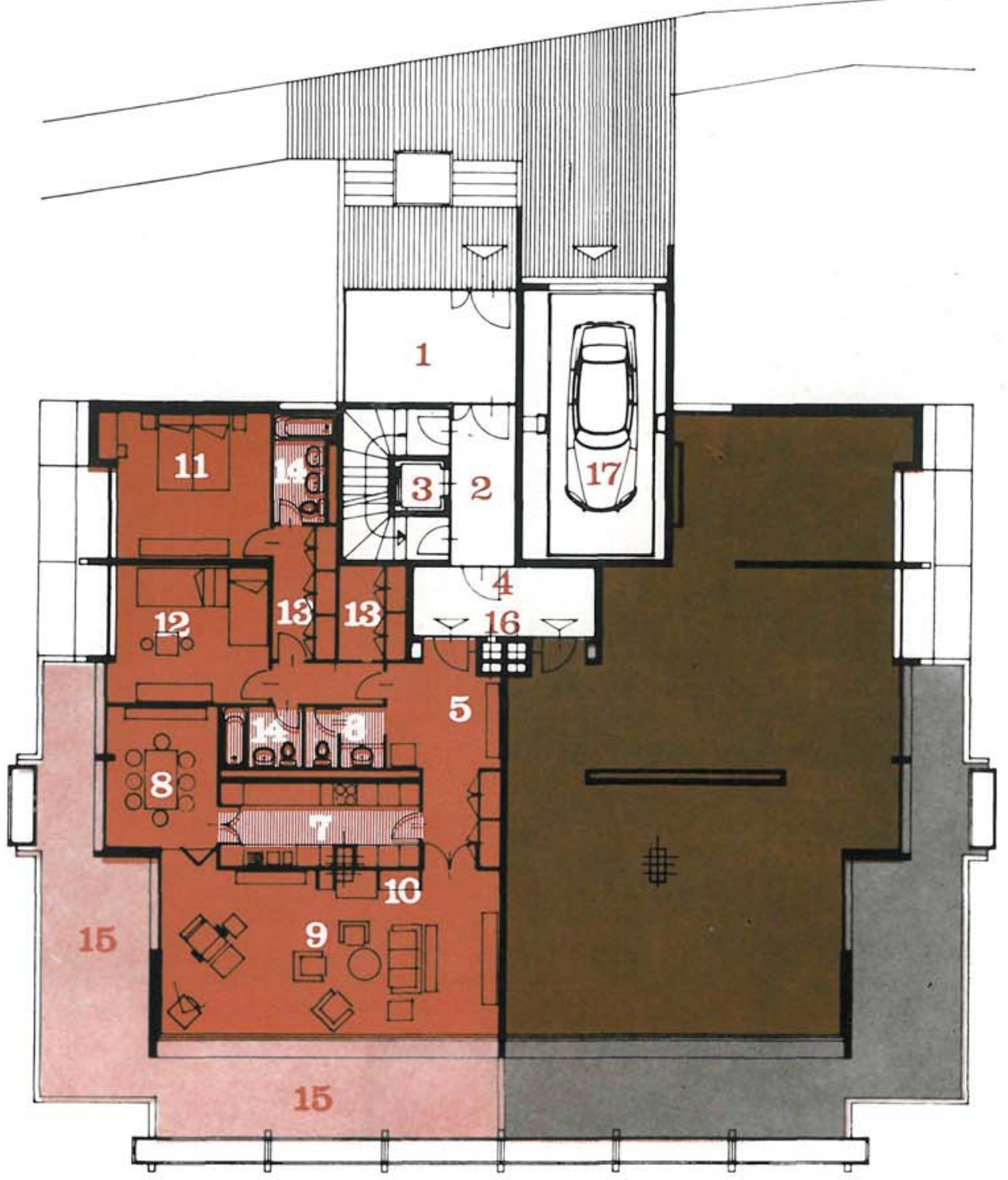

\section{tercera}

cuarta
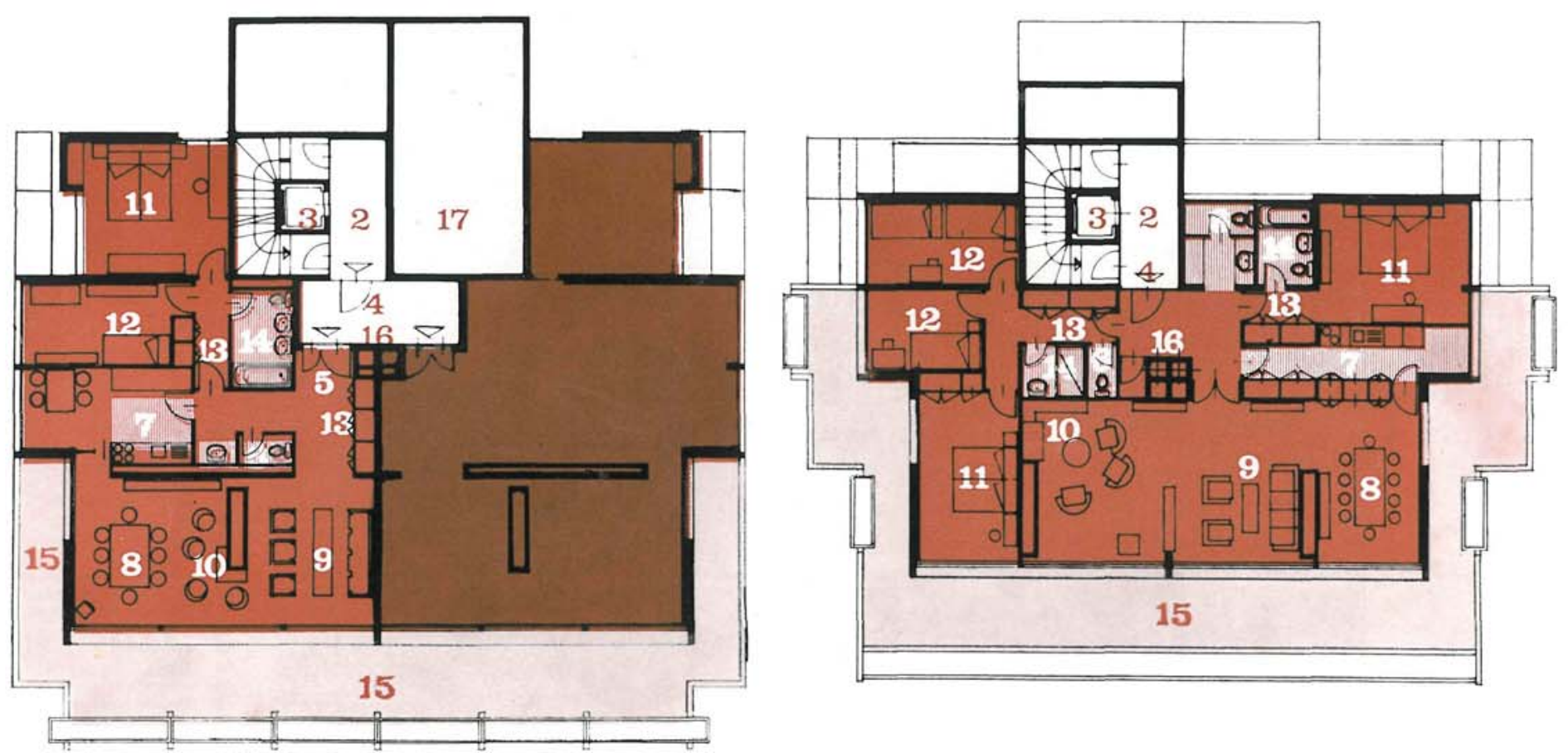
El programa inicial señalaba la construcción de un edificio de tipo residencial, con abundancia de terrazas asomándose sobre el lago, aprovechando su orientación sur. Al mismo tiempo debía estar adaptado al máximo a la naturaleza del terreno.

En lo que respecta a su distribución interior, el único condicionante era que las superficies destinadas a servicio fueran muy restringidas.

Las dimensiones del edificio, a su vez, venían definidas por su alineación con el borde del lago y por la altura y volumen máximos permitidos.

La solución adoptada consistió en un edificio en forma de pirámide escalonada por tres de sus caras, lo que proporcionaba una gran superficie de terrazas en cada planta, aprovechando el techo de la inferior, al tiempo que se establecía un paralelaje con la inclinación del terreno. Las terrazas, profusamente ajardinadas, acentuarían la incorporación del edificio al paisaje de alrededor.

La construcción consta de cinco plantas y sótano. En este último se alojan todos los locales técnicos y los servicios comunes, así como trece plazas individuales de garaje y otras más para pequeñas embarcaciones que acceden directamente desde el lago. En los cinco niveles superiores se distribuyen 13 apartamentos de diferentes formas y tamaños, que varían desde el pequeño estudio de una sola pieza hasta el apartamento más grande de seis habitaciones, situado en el nivel superior. Rematando el edificio se encuentra una amplia terraza, que sirve de jardín y solario para este último apartamento.

La forma piramidal del edificio trajo consigo distintas dificultades:

- Una de ellas en la distribución interior de los apartamentos, principalmente en los niveles inferiores, ya que a las grandes superficies del centro de la planta difícilmente podía llegar la iluminación natural. Esto obligó a la búsqueda de una distribución adecuada, a base de grandes espacios abiertos comunicados entre sí, que pusieran el mínimo de impedimentos a la luz que entraba por los amplios ventanales de las fachadas.

- Otra en el acceso de los vehículos al inmueble. En un principio se pensó en efectuarlo por el sitio más cómodo, dada la situación de los garajes, es decir, por la orilla del lago, pero estaba prohibido, lo que motivó su emplazamiento en la tercera planta del edificio, a nivel de la calle superior. La diferencia de alturas, y la escasa superficie, que no permitía rampas, obligaron a la instalación de un montacargas especial para automóviles, que los transportara desde el nivel de entrada hasta el sótano.

- La inclinación del edificio supuso también una cierta dificultad en la distribución de las conducciones verticales, tanto de las chimeneas como de las canalizaciones y tuberías de agua y calefacción. Esto se solucionó agrupando todos los conductos en la parte interior del edificio, y dejando el exterior, siempre que fuese posible, libre de instalaciones de servicio.

En la construcción general del edificio se ha empleado hormigón armado. La cimentación se realizó mediante una losa de hormigón armado. La estructura emplea pilares y muros arriostrados, concebidos de tal manera que permiten una gran libertad en la distribución interior de los distintos apartamentos.

Exteriormente predomina el hormigón visto en el conjunto de la obra, revestido con estuco de cemento blanco coloreado. Las fachadas laterales se hicieron con elementos prefabricados de hormigón. La fachada sur consta, por una parte, de una serie de jardineras de diferentes tamaños y formas, con sus soportes, todo ello prefabricado con hormigón liso;

el resto, que hace juego con las jardineras, es de hormigón coloreado, vertido en obra, para el que hubo que emplear un sistema de encofrados muy complejo, dada la composición del diseño arquitectónico. En la fachada norte se ha seguido el mismo principio que en las otras, con la diferencia del elemento decorativo, en cerámica de color, dispuesto en el frontón de entrada. Estos materiales se eligieron tanto por su nobleza como por su gran resistencia, que no varía con el transcurso del tiempo. 
Los grandes ventanales que dan sobre las amplias terrazas unen, a la satisfacción de un interior lujoso y confortable, el placer de la vida al sol.

Toda la carpintería exterior está formada por bastidores forrados de aluminio.

El suelo del vestíbulo de entrada es de mármol, mientras que el de los corredores que en cada planta dan acceso a los apartamentos, va revestido con moquetas,

al igual que los suelos de estos últimos.

Las cocinas, cuartos de baño y aseos están equipados con todo tipo de modernos sanitarios y elementos de servicio, con alicatados de cerámica decorativa, de color, en los paramentos verticales.
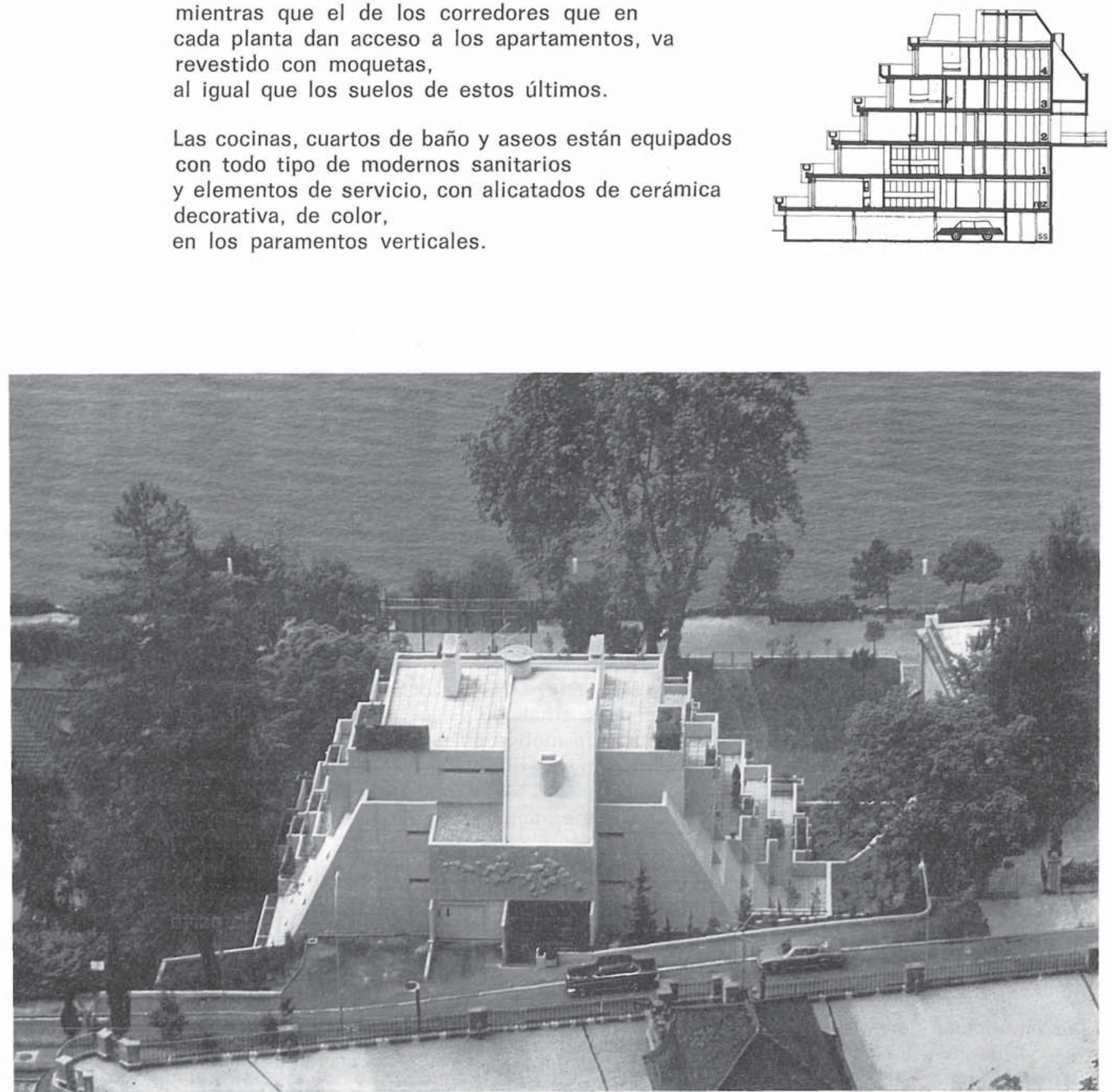

Fotos: HENRIETTE GRINDA

Como instalaciones el edificio cuenta con:

- Una central térmica de fuel-oil y una cisterna exterior de 30.000 litros de capacidad. El agua caliente producida es llevada por monotubos situados bajo el suelo.

La calefacción se consigue mediante radiadores, convectores y paneles radiantes.

Este sistema, equipado con reguladores y termostatos exteriores,

garantiza un funcionamiento regular y racional durante todo el año. 
- Un sistema de ventilación mecánica para las cocinas, cuartos de baño y aseos.

El garaje cuenta con una instalación independiente.

- Un ascensor rápido para cuatro personas, lujosamente equipado, sirve todas las plantas, incluyendo el sótano.

- Un montacoches, de dimensiones suficientes para transportar los automóviles más grandes, asegura la unión entre la calle y el garaje del sótano.

- Un sistema de portero electrónico, accionable desde cada apartamento.

- Una instalación de teléfono y antenas de televisión y frecuencia modulada, en cada apartamento.

- Un sistema de acondicionamiento térmico y acústico, esencial para un buen confort, ha sido cuidadosamente estudiado.

— Y, por último, un conducto para eliminación de basuras.

Con toda esta variada serie de instalaciones y servicios, cada apartamento, funcional y espacioso, ofrece las ventajas de una vivienda individual, con el confort de un edificio moderno.

\section{résumé}

Edifice d'habitations à Montreux Suisse

Jean Serex, architecte SIA

Construit sur un terrain, près du lac, sur la promenade des Fleurs de Territet, cet édifice adopte la forme de pyramide tronquée, avec un sous-sol pour les installations et les garages, et cinq niveaux réservés aux appartements.

Fondations spéciales, ossature en béton armé et murs extérieurs à base d'éléments divers préfabriqués.

L'auteur du projet a étudié les nombreux problèmes posés par las Ordonnances de la zone, la forme de l'édifice et l'adapta. tion au terrain et a abouti à un résultat tion au terrain et a abouti à un résultat extrêmement satisfaisant à tout
gences résidentielles prévues.

\section{summary}

Apartment building in Montreux Swizerland

Jean Serex, architect SIA

This building has been constructed on a beautiful plot next to the lake in the Flower Avenue in Territet. It assumes the shape of a truncated pyramid, with a subterra. nean floor for installations and garages and 5 apartment stories.

Special foundation, structure of reinforced concrete and curtain walls consisting of concrete and curtain walls consisting of rous problems caused by the laws of the area have been studied and solved success. fully, as well as the shape of the building and the adaptation to the terrain. Thus an extremely satisfactory result has been obtained in view of the residential requirements.

\section{zusammenfassung}

Wohnungsgebäude in Montreux Schweiz

Jean Serex, Architekt SIA

Dieses Gebäude ist auf einem hübschen Grundstück in der Nähe von dem See auf der Blumenpromenado in Territet gelegen. Es nimmt die Form einer abgestumpften Py. ramide an, mit einem unterirdischen Stock. Stockwerken mit Wohnungen.

Spezialgründung. Struktur aus Stahlbeton und Umschliessung auf Grund von etlichen vor. gefertigten Elementen.

Die vielen Probleme, von den Verordnungen der Gegend verursacht, sind sehr gelunge studiert und gelöst worden, wie auch die Form des Gebaudes und das Anpassen an den Boden und man hat dadurch ein Ergeb. nis bekommen, das ausserordentlich befrie. digend für alle vorgesehene Wohnforderungen war. 